\title{
Wound Closure: Evidence of Cooperation Between Fibroblasts and Collagen Matrix
}

\author{
H. P. EHRLICH
}

Boston

\begin{abstract}
Summary
The closure of severe wounds where viable tissue has been destroyed by trauma involves the depositing of a new connective tissue matrix, the amount of which is dictated by the severity of trauma. That new connective tissue matrix is immature, and in some cases, can reduce itself. When this occurs in a healing wound it is called wound contraction. When it occurs in the scar of a healed wound, it is called scar contracture. Forces generated in fibroblasts organise the surrounding connective tissue matrix, and this is responsible for the contraction of wounds and the contracture of scars. The experimental work presented here supports the idea that these contractile forces residing in the fibroblast work as individual units to contract the wound. There is no evidence to support the idea of the myofibroblast, a specialised cell, being responsible for contractile forces, as reported. The morphological appearance of stress fibres, which denote the presence of myofibroblasts, may, in fact, signify the termination of the generation of contractile forces. Control of cellular contractile forces appears to be linked to the composition of the connective tissue matrix. A matrix rich in type III collagen contracts faster and to a greater degree than one made from type I collagen. It is suggested that granulation tissue and immature scar with a matrix rich in type III collagen will contract more readily than a more mature scar with less type III collagen. Evidence presented from in vitro models suggests that fibroblasts generate the forces of contraction, and collagen controls those forces in wound closure and scar contracture.
\end{abstract}

Healing of dermal wounds requires the synthesis of a new connective tissue matrix. The amount of new connective tissue deposited depends on the size and depth of the trauma. For example, a sutured surgical incision requires a small amount of new connective tissue to mend itself completely. A third degree burn, in contrast, left to close on its own, requires much more new connective tissue for repair and closure.

Open wounds in such laboratory animals as rats will close by the combination of the deposition of a new connective tissue matrix and wound contraction. The latter involves the drawing of surrounding skin, dermis and epidermis, over the defect by forces residing in the granulation tissue. The skin does not regenerate; instead, the dynamic movement of the skin surrounding the defect covers it. Wound contraction is not a major contribution to wound closure in most parts of the the human body. The defect is initially filled with granulation tissue which eventually matures to scar and wound contraction is minimal. A severe injury will, therefore, be covered by a scar tissue patch which can be unstable and undergo scar contracture resulting in distortion of surrounding tissue. The 
surgeon will graft third degree burns to reduce the amount of scar required to cover the defect, and this, in turn, reduces the amount of scar contracture. The major event in grafting is the acceptance of the graft, and wound healing is limited to the space between the host and the graft. Because of this, the amount of new connective tissue deposited is minimal, and scarring is restricted to the edges of the graft.

Human scar can have traumatic cosmetic and functional effects. When it occurs over a joint, it can lead to contractures which impair the motion of that joint. The connective tissue of scar can be slow to mature, and this can lead to a reduction known as scar contraction. In this case, contractile forces are generated from within the scar tissue. These forces cause that tissue to become compact, and this can force a joint into flexion, thereby hindering extension of that joint. ${ }^{1}$ In most cases, a rigorous and lengthy program of physical therapy is necessary to eliminate the contracture brought about by these contractile forces.

The forces of wound contraction and scar contracture appear to reside in the cellular components of the connective tissue matrix of the healing or healed injury. It is a popular notion that the myofibroblast, a specialised cell, generates these forces. ${ }^{2}$ That theory is based on the morphological identification of these special cells in contracting tissues. ${ }^{3}$ The myofibroblast has cytoplasmic stress fibres which are rich in actin. It is proposed that when these muscle-like fibres contract, they contract the entire cell. Because myofibroblasts adhere to one another, this sheet of contracting cells acts like a multi-cellular muscle. This multi-cellular contractile unit draws the surrounding connective tissue matrix into a new position. This theory of wound closure and scar contracture is based on a hypothesis of an integrated multi-cellular unit undergoing cell contraction.

Another explanation of contractile forces generated in wound closure and scar contracture is that fibroblasts work as individual cellular units to reorganise the surrounding connective tissue matrix. The forces which cause connective tissue to reorganise are the same ones which generate cell locomotion.
According to Harris and co-workers, cell locomotion does not occur by cell contraction, but by tractional forces whereby the cell retains a spread and elongate shape while the periphery engages in a motion similar to a tank tread. ${ }^{4}$

Cell contraction, the shrinking of the entire cell, by cytoplasmic stress fibers can be demonstrated in vitro. Human dermal fibroblasts cultured on glass coverslips are permeabilised with gylcerol. ${ }^{5}$ When a solution of ATP with metal co-factors is added to these treated cellular preparations, the cells shrink, that is, they contract. Using rhodamine phalloidin (Rh-phalloidin), a stain specific to actin filaments, stress fibres, rich in actin, are seen in fibroblast preparations. ${ }^{6}$ After the addition of ATP and the resultant cell contraction, these actin rich filaments take the form of compact aggregates. ${ }^{5}$ This is the result of the sliding mechanism of actin-myosin filaments. Conditions which prevent the myosin ATPase activity required to produce that sliding will inhibit the cell contraction induced by adding ATP. ${ }^{5}$

Polyvinyl sponges were subcutaneously implanted in rats to test for cell contraction in granulation tissue. ${ }^{7}$ They were removed on day 7 and processed for cryosectioning. Frozen sections were treated with glycerol and their cytoplasmic stress fibres were displayed by Rh-phalloidin staining (Fig. 1a). Some of these permeabilised sections were treated with ATP and co-factors for $10 \mathrm{~min}$ before staining (Fig 1b). Granulation tissue appears to have a dense cellular population which readily undergoes cell contraction when ATP is present. This shows that cells in granulation tissue have myosin ATPase activity which contributes to the aggregation of cytoplasmic stress fibres.

An in vitro model which demonstrates cell locomotion is that of silicone oil thin-film surface wrinkling introduced by Harris and co-workers. ${ }^{4}$ Figure 2 a shows human dermal fibroblasts wrinkling a thin film surface. Those fibroblasts, stained for actin rich stress fibres, exhibit none at 24 hours (Fig. 2b). At 48 hours, when new wrinkling is almost non-existent, the same fibroblasts demonstrate cytoplasmic stress fibres (Fig. 2c). It appears that cell locomotion generates surface wrin- 
a.
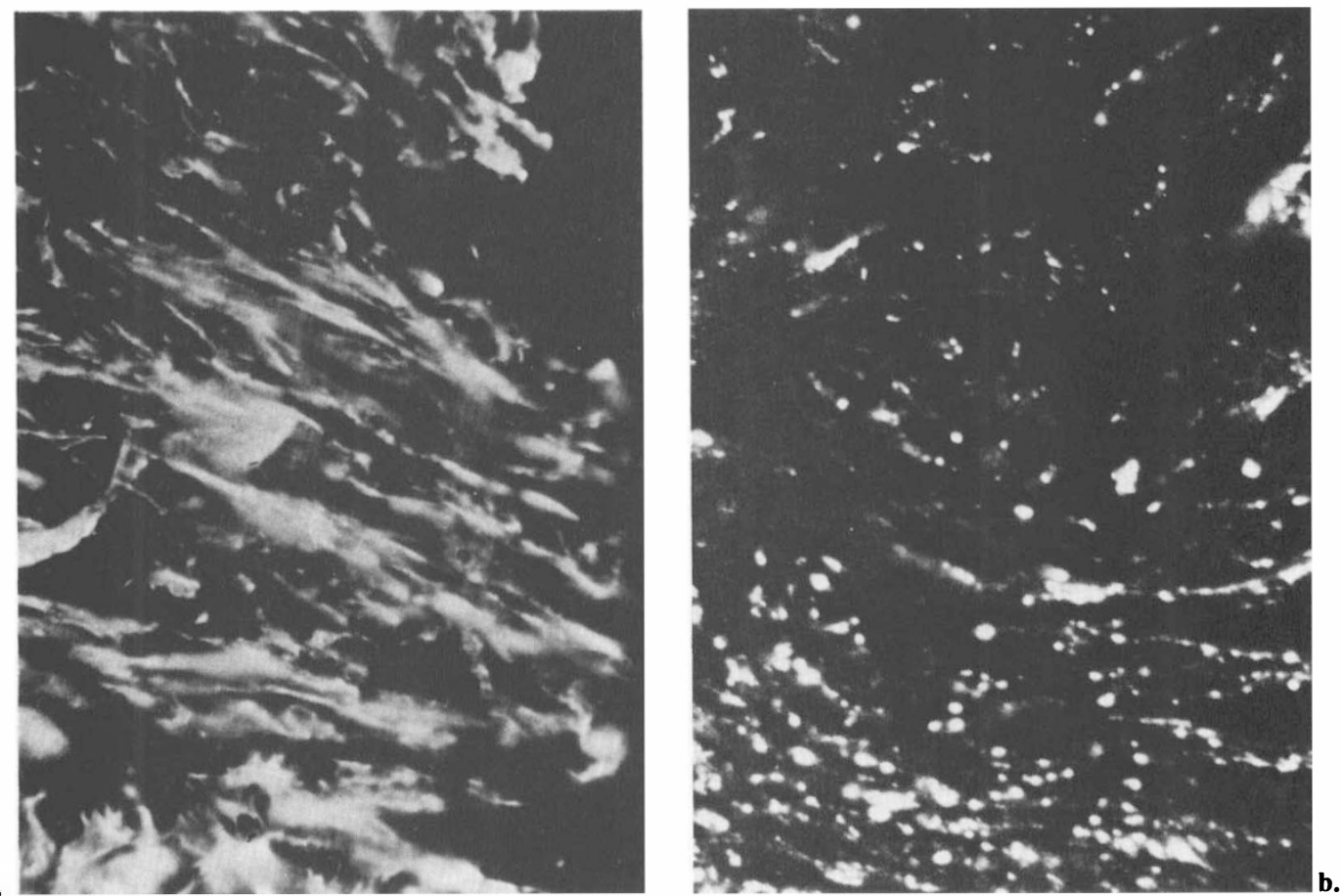

Fig. 1. Polyvinyl sponge disc implants $1.5 \mathrm{~cm}$ in diameter and $0.2 \mathrm{~cm}$ thick were subcutaneously placed in adults rats $350 \mathrm{gm}$. The sponges were dissected out at 7 days and prepared for cryosectioning. The $6 \mu \mathrm{m}$ thick section was treated with glycerol and stained with Rh-phalloidin before or $10 \mathrm{~min}$ after the addition of ATP. Histological sections were viewed and photographs were made using epifluorescence. ${ }^{5}$

(a). Rh-Phalloidin stained section of sponge granulation tissue shows actin-rich cytoplasmic staining after being treated with glycerol and before the addition of ATP.

(b). Rh-phalloidin section of similar preparation 10 min after the addition of $1 \mathrm{mM}$ ATP and metal co-factors.

kling by tractional forces in the absence of stress fibres. Surface wrinkling occurs in the absence of cell contraction. When tractional forces become static, and cell locomotion has ceased and there is no further surface wrinkling, cytoplasmic stress fibres appear. Hence, during the period of the generation of cellular forces, stress fibres are absent. They appear only after active cell locomotion.

The in vitro study of wound contraction and scar contracture employs a model system first described by Bell and co-workers. ${ }^{8}$ The fibroblast populated collagen lattice (FPCL) allows the investigation of the interaction of fibroblasts and collagen in a dermis-like matrix shrinking in the process knows as lattice contraction. When fibroblasts, freed from monolayer cultures by trypsin, are mixed in a petri dish with culture medium containing serum and native collagen, the collagen poly- merises and traps the cells in the collagen matrix. This FPCL eventually undergoes a reduction directly proportional to cell number and inversely proportional to collagen concentration. Fibroblasts and smooth muscle cells are highly efficient at lattice contraction..$^{8,9}$

During lattice contraction fibroblasts tend to be elongated and spread. Additives which induce high intracellular levels of cAMP alter cell morphology and render them rounder and less efficient at contracting a lattice. ${ }^{10}$ In the first 24 hours after manufacture of the FPCL, the actin filaments of the fibroblasts contained therein appear to have a fine filamentous structure. As the FPCL contracts from an initial area of $800 \mathrm{~mm}^{2}$ to $50 \mathrm{~mm}^{2}$ at 48 hours, fibroblasts display few cytoplasmic stress fibres (Fig. 3a). At 96 hours, however, when lattices have contracted to $30 \mathrm{~mm}^{2}$, there is 


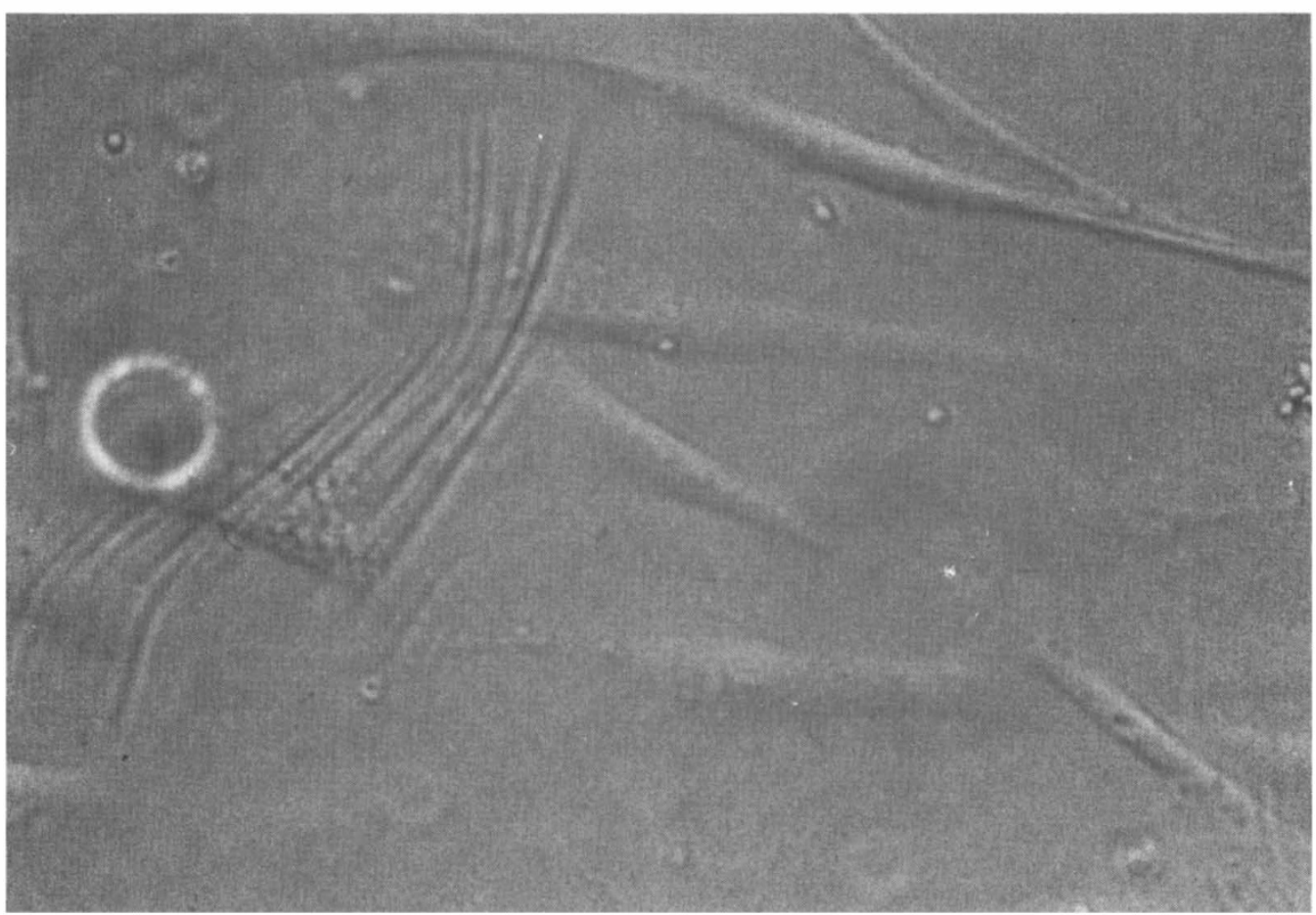

Fig. 2a.

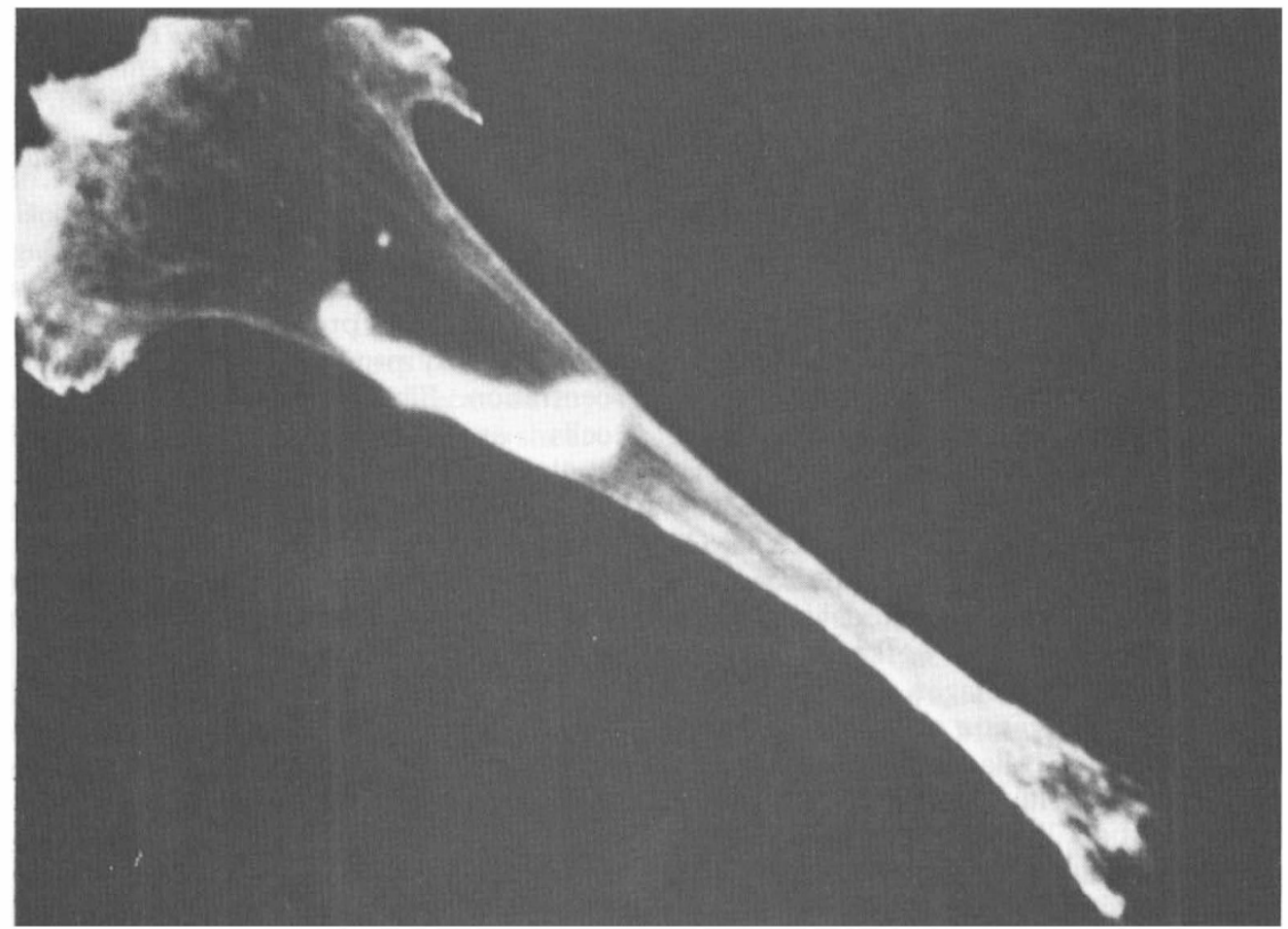

Fig. 2b. 
Fig. 2c.

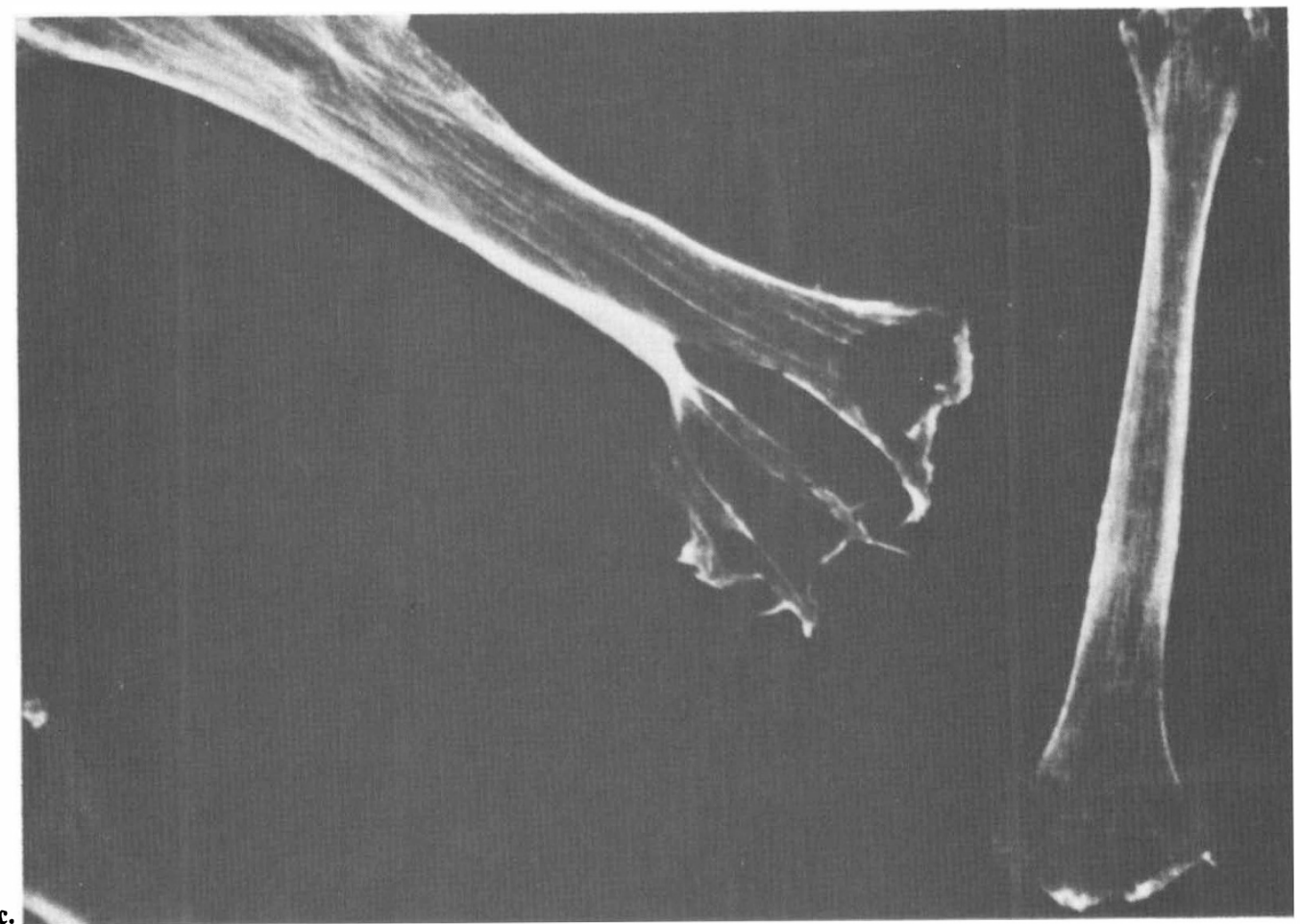

Fig. 2. Normal human fibroblasts plated onto a thin film of polymerised silicone oil. ${ }^{4}$

(a). Four hours after plating some fibroblasts are shown, under phase contrast optics, to wrinkle the surface beneath them.

(b). A similar preparation fixed and stained with Rh-phalloidin 4 hours after plating. Actin is present at the leading edge of the fibroblast where wrinkling is occurring.

(c). Fibroblasts fixed and stained with Rh-phalloidin shown 48 hours after plating. Stress fibres are present in the cytoplasm of the cell when no new wrinkling is occurring.

little contraction and prominent stress fibres are evident. During rapid lattice contraction, therefore, cells are elongate and have few stress fibres. When contraction slows or is absent, cytoplasmic stress fibres are prominent (Fig. 3b). This implies that fibroblasts are responsible for lattice contraction. Myofibroblasts develop in FPCL only when there is much cell-cell contact and the rate of lattice contraction is minimal. ${ }^{11}$

Fibroblasts are found in high density at the periphery of the lattice during the early stages of contraction. The reason for this is the reorganisation of the matrix at the periphery of the lattice which results in the collapse of the matrix and less space between cells. Cells in contact with each other have a myofibroblast morphology with cytoplasmic stress fibres. Since the outer edge develops myofibroblasts faster than other areas of the lat- tice, the relationship between lattice contraction and myofibroblasts in the periphery of the lattice can be studied. The multicellular unit of synchronised cell contraction as the driving force of lattice contraction, based on the myofibroblast theory, ${ }^{12}$ can be investigated in the FPCL. Six hours after casting, lattices are divided into two groups. Those in the first group have a circular hole punched from the middle and the others remain intact (Fig. 4a). Lattices with holes have a doughnut-like configuration with a dense ring of cells having many cell-cell contacts at the outer edge. The intact lattices have the same peripheral ring of cells, but they also contain more cells and collagen in the central region. The doughnut-like lattice has greater proportions of myofibroblasts than the intact lattice. At 24 hours, the lattices are of identical size (Fig. 4b). Increasing the proportions 


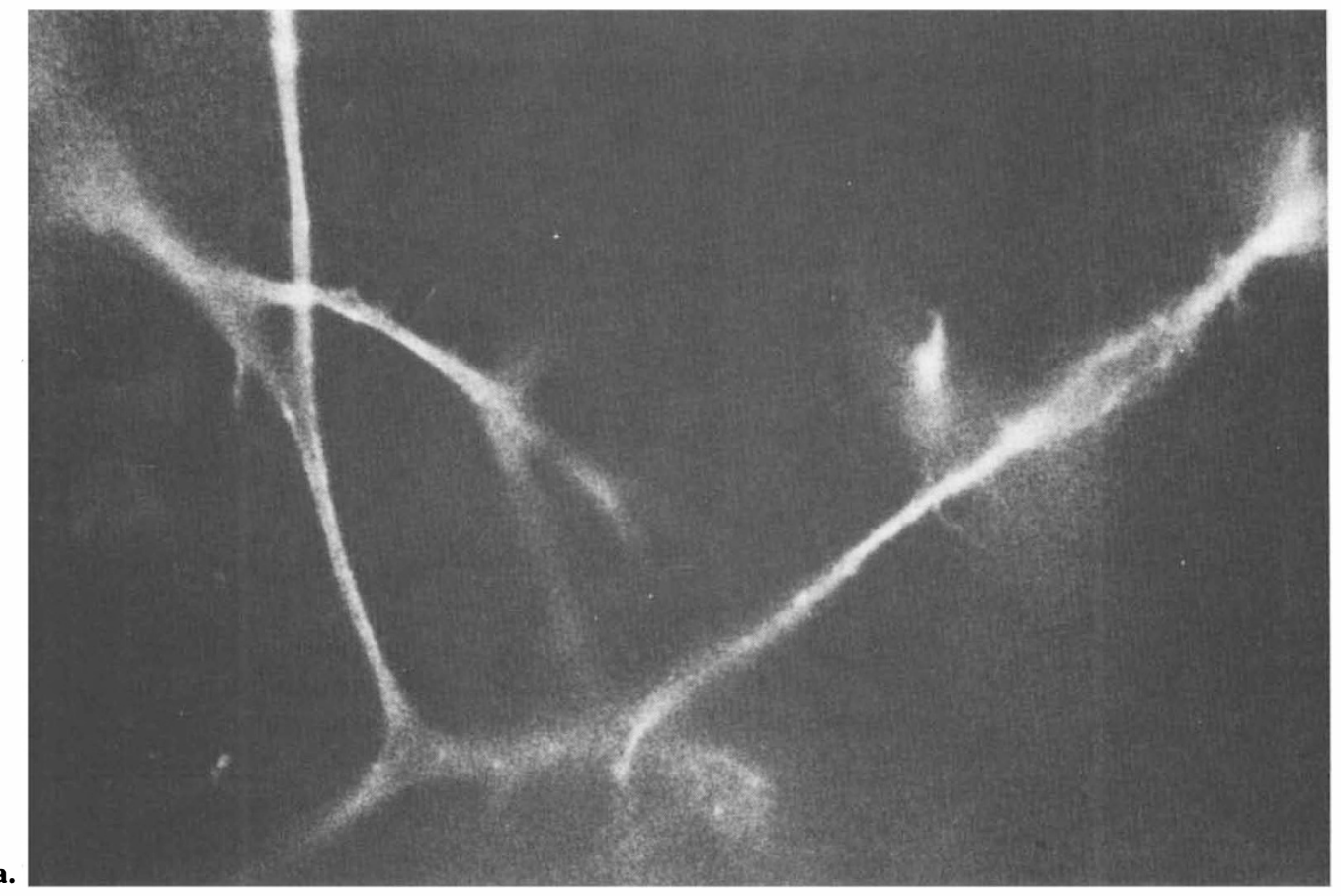

a.

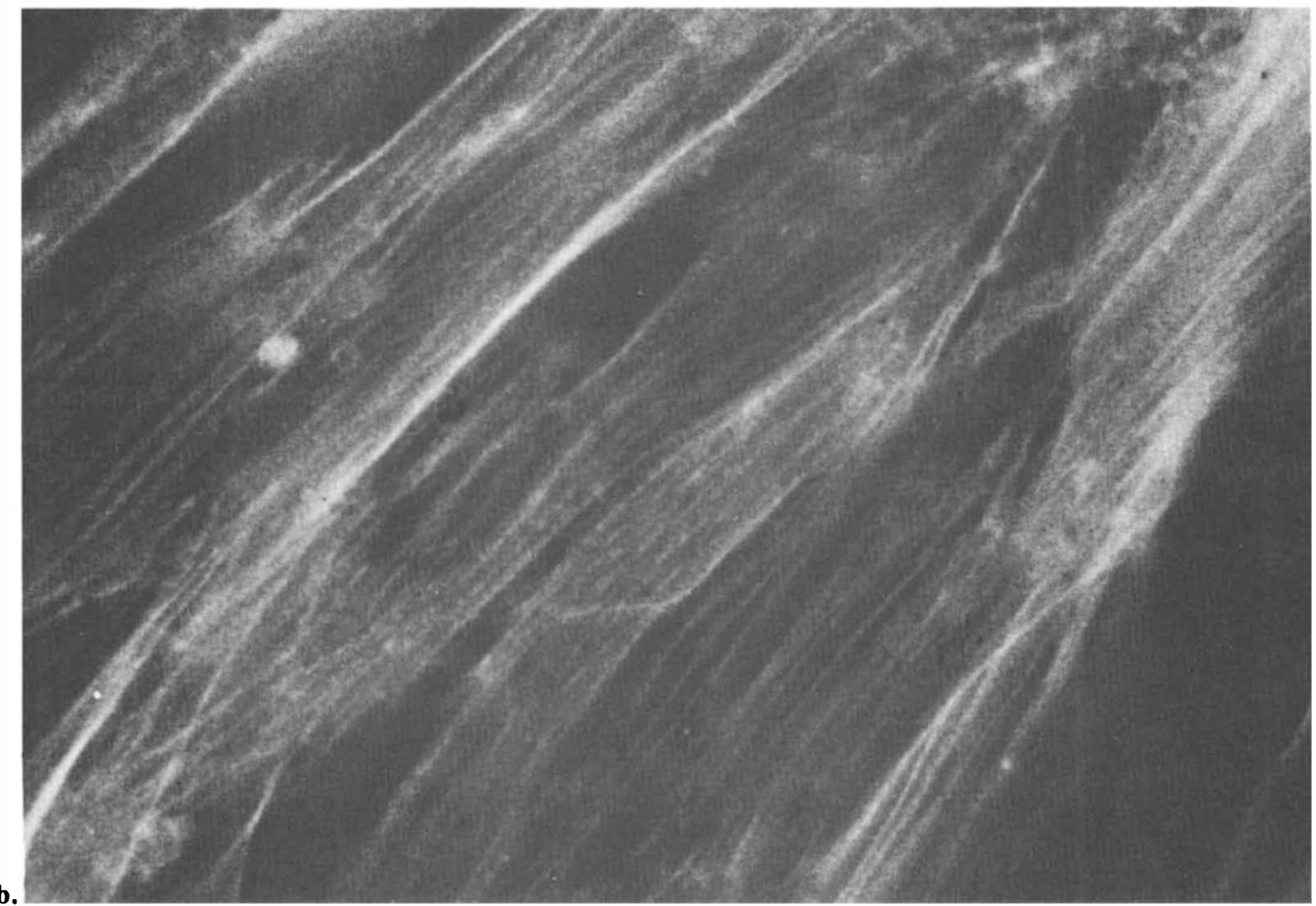

Fig. 3. Fibroblast populated collagen lattices manufactured and fixed and stained with Rh-phalloidin. They were viewed and photographed using epifluorescence.

(a). Fibroblasts in the centre of the lattice 24 hours after manufacture are surrounded by collagen and show diffuse staining throughout the cytoplasm.

(b). Fibroblasts are shown 96 hours after manufacture of an FPCL when no further lattice contraction is evident. They are found at the edge of the lattice and have many cell-cell contacts and prominent stress fibres. Morphological evidence determines that they are myofibroblasts. 
a.

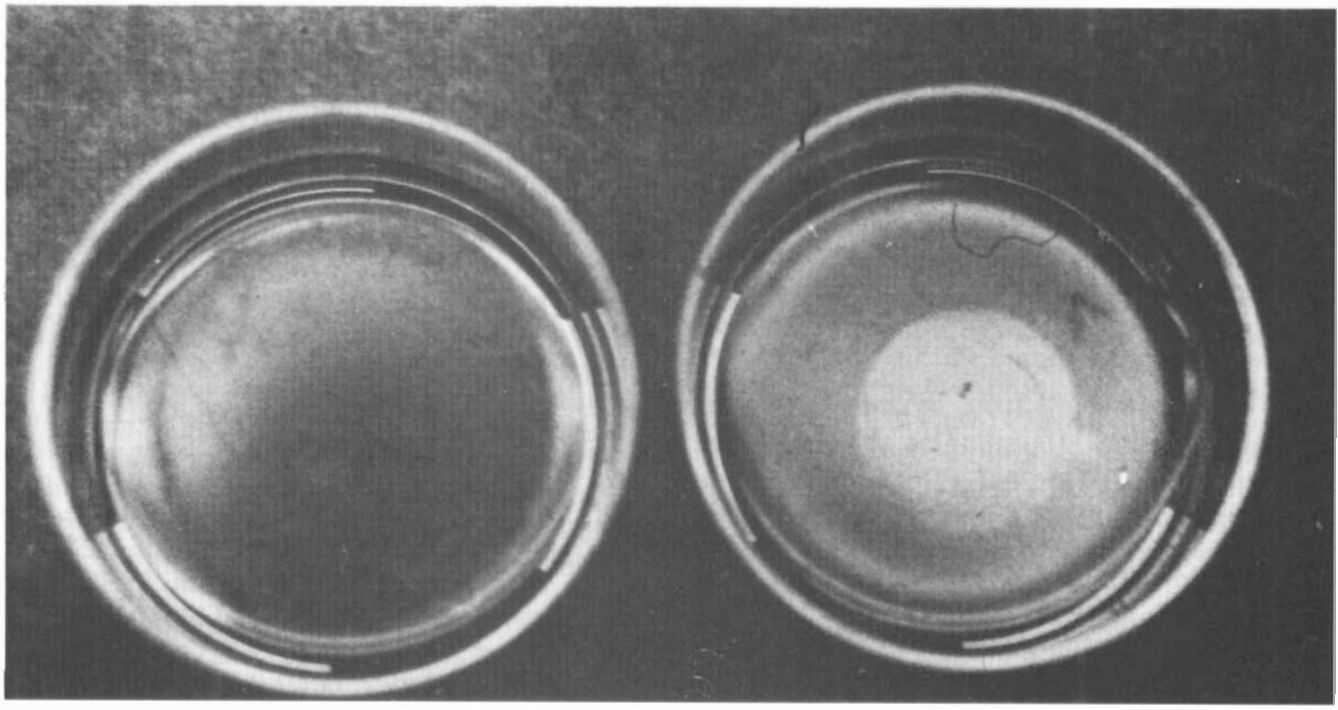

b.

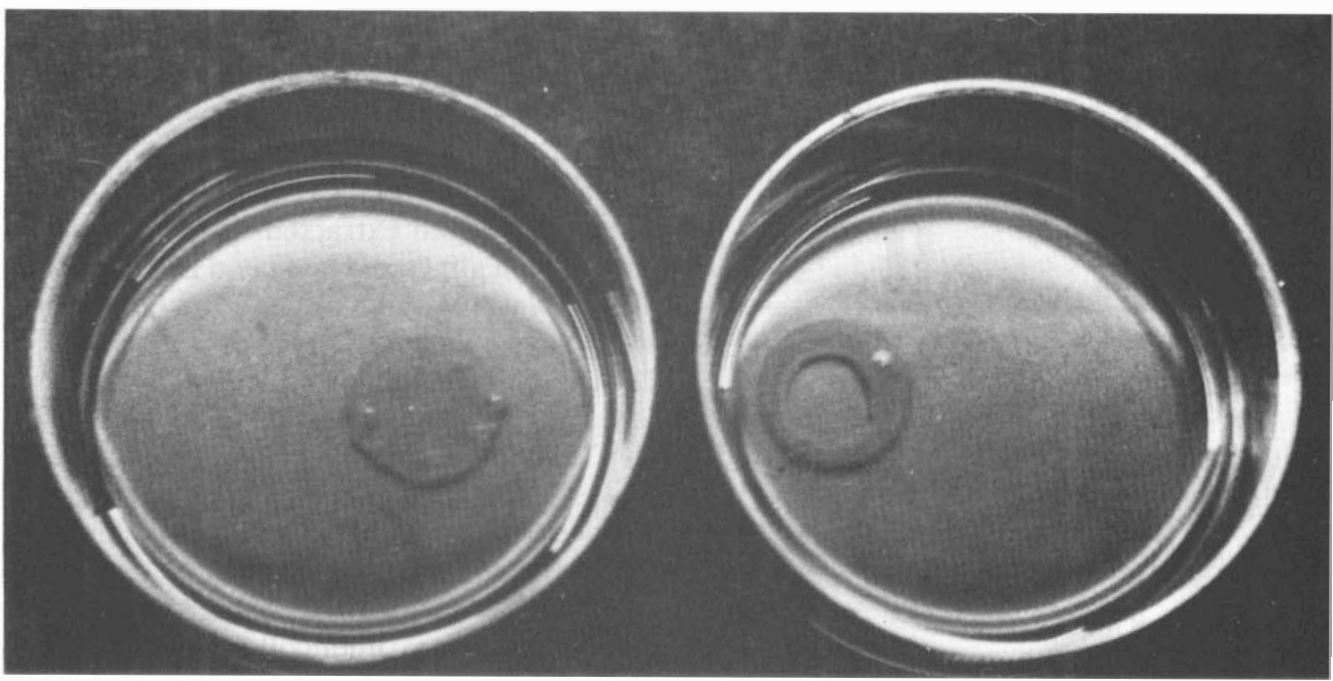

Fig. 4. FPCL were manufactured and a hole was punched in some at 6 hours when contraction had just begun. $A$ dense ring of cells can be seen in the edge of the lattice in Figure $4 a$. Figure $4 b$ shows, 18 hours later, that lattice contraction has proceeded. The output diameters of the lattices are equal, indicating that lattice contraction is equal in both.

of myofibroblasts in the FPCL does not enhance lattice contraction. The results from this experiment support the idea that cells work individually to organise the collagen matrix.

The role of collagen in FPCL contraction was studied using pepsin-solubilised collagen extracted from human tissue..$^{13}$ Two groups of FPCL were manufactured under identical conditions, and fibroblasts were grown out from normal human skin or hypertrophic scar. FPCL with identical numbers of cells demonstrated no variation in size over time (Fig. 5a). The source of the fibroblast had no influence on the rate of lattice contraction. Hypertrophic scar, where contracture occurs, gives no evidence of a specialised contractile cell residing therein.

If equal concentrations of collagen are used to make FPCL under identical conditions, 
with one group of FPCL made from collagen from normal human skin and the other from collagen from hypertrophic scar, differences are evident. FPCL made with collagen from hypertrophic scar contracted faster and to a greater degree than lattices with collagen from normal skin (Fig. 5b). Since the manufacturing process was identical in each group, it seems that the source of collagen influences lattice contraction. ${ }^{11}$

Collagen extracted from hypertrophic scar is richer in type III collagen than collagen from normal dermis. ${ }^{14,15}$ FPCL were made from type I, II, or III collagens to determine

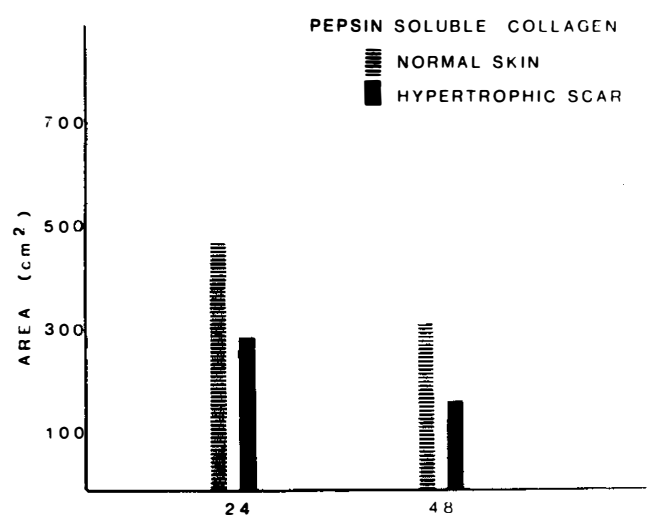

a.

TIME IN HOURS

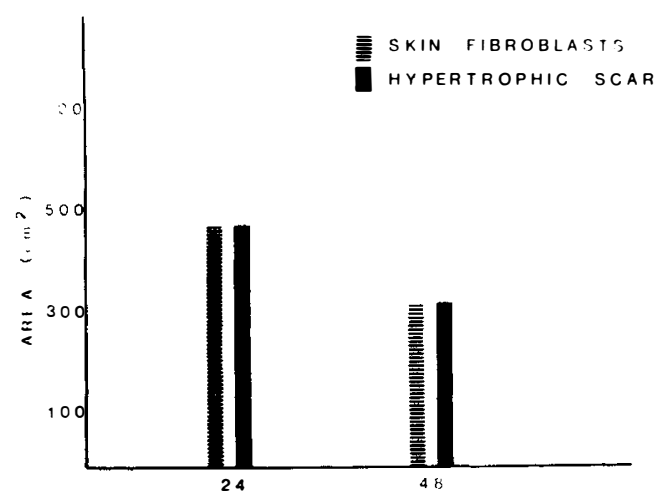

b.

Fig. 5. Graphic presentation is made of the results from FPCL contraction.

$A$. Lattices made under identical conditions using cells from normal human dermis or from hypertrophic scar. Each had identical abilities to contract the lattice.

$B$. Lattices made under identical conditions with collagen pepsin-extracted from normal dermis or from hypertrophic scar. Lattices made from hypertrophic scar contracted faster and to a greater degree than the others.

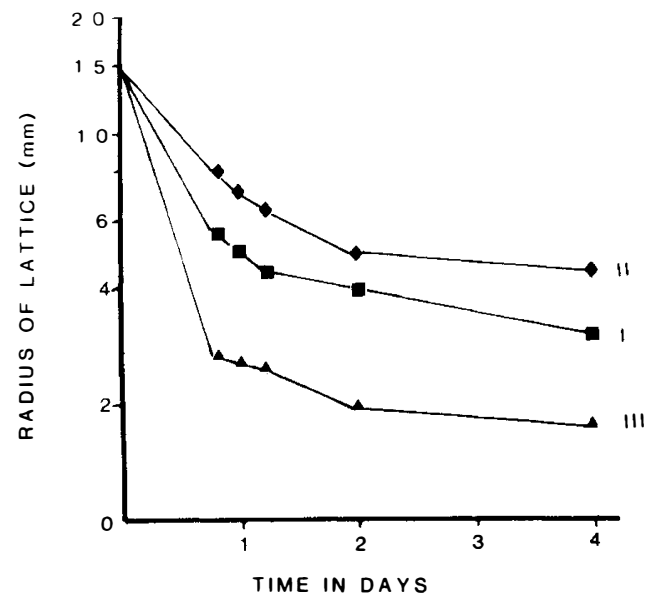

Fig. 6. FPCL were made under identical conditions using type I, II or III collagen extracted from normal human dermis, cartilage, or leiomyoma. Those made using type III collagen contract faster and to a greater degree than those made from other types.

whether collagen type would influence lattice contraction. Type I collagen was extracted from normal skin and isolated from other collagen types by solubilising it in $1.7 \mathrm{M} \mathrm{NaCl}$. Type II collagen extracted from human cartilage was a gift from Dr. David Eyre, University of Washington, Seattle. Type III collagen was pepsin-extracted from a leiomyoma, a benign uterine tumor, and was further isolated by its insolubility in $1.7 \mathrm{M} \mathrm{NaCl}$. All FPCL contained $1.25 \mathrm{mg}$ collagen per ml lattice. Figure 6 presents the differences between the rates of lattice contraction and ultimate size. Lattices made from type III collagen contract faster and to a greater degree than lattices from other collagen types. FPCL of type II collagen contracted the slowest and the least.

In summary, wound contraction and scar contracture occur by forces produced by resident cells. Evidence presented supports the idea that most fibroblasts are equal in their ability to produce forces to contract wounds or distort scars. It appears that the residual matrix of the closing wound or scar may be important in controlling the contractile process. Modulating the connective tissue matrix of fibrotic tissue may be a direction for altering scar contractures. There is little evidence to support the role of the myofibroblast, a specialised cell, in these contractile processes. 


\section{References}

${ }^{1}$ Brody GS, Peng TJ, Landel RF: The etiology of hypertrophic scar: Another view. Plast Reconst Surg 1984; 67: 673-84.

${ }^{2}$ Gabbiani G, Hirschel BJ, Ryan GB, Statkov PR, Majno G: Granulation tissue as a contractile organ: A study of structure and function. J Exp Med 1972; 135: 719-34.

${ }^{3}$ Hirschel BJ, Gabbiani G, Ryan GB, Majno G: Fibroblasts of granulation tissue: Immunofluorescent staining with anti-smooth muscle serum. Proc Soc Exp Biol Med 1971, 138: 466-9.

${ }^{4}$ Harris AK, Wild P, Stopak D: Silicone rubber substrate: A new wrinkle in the study of cell locomotion. Science 1980, 280: 177-9.

${ }^{5}$ Ehrlich HP, Rajaratnam JBM, Griswold TR: ATP induced cell contraction in dermal fibroblasts: Effects of cAMP and myosin light chain kinase. $J$ Cell Physiol 1986, 128: 223-30.

${ }^{6}$ Weiland T and Faulsltich H: Amatoxins, phallotoxine, phallolysin and atamanide: the biologically active components of poisonous Amanita mushrooms. CRC Crit Rev Biochem 1978, 5: 185-260.

${ }^{7}$ Ehrlich HP and Traver H: Effects of Beta-Carotene Vitamin A, and glucocorticoids on collagen synthesis in wounds. Proc Soc Exp Biol Med 1971, 137: $936-8$

${ }^{8}$ Bell E, Ivarsson B, Merrill C: Production of a tissuelike structure by contraction of collagen lattice by human fibroblasts of different proliferative potential in vitro. Proc Natl Acad Sci (USA), 1979 76: 1274-8.

${ }^{9}$ Ehrlich HP, Griswold TR, Rajaratnam JBM Studies of vascular smooth muscle cells and dermal fibroblasts in collagen matrices: Effects of heparin. Exp Cell Res 1986, 164: 154-62.

10 Ehrlich HP and Griswold TR: Epidermolysis Bullosa dystrophica recessive fibroblasts produce increased concentrations of cAMP within a collagen matrix. J Invest Derm 1984 83: 230-3.

11 Ehrlich HP: The role of connective tissue matrix in hypertrophic scar contracture. In Hunt TK, Heppenstall RB, Pines E, Rovee D eds Soft and Hard Tissue Repair: Biological and Chemical Aspects. New York: Praeger 1984: 533-53.

12 Majno G, Gabbiani G, Hirschel BJ, Ryan GB: Contraction of granulation tissue in vitro: Similarity to smooth muscle. Science 1971, 173: 548-50.

${ }^{13}$ Ehrlich HP: The connective tissue matrix control of cell mediated wound contraction and scar contracture. J Trauma 1984; 24: S35-39.

${ }^{14}$ Bailey AJ, Bazin S, Sim TJ, LeLous M, Nicoletis C, DeLaunay A: Characterisation of the collagen of human hypertrophic and normal scars. Biochem Biophys Acta 1975; 405: 412-21.

${ }^{15}$ Bailey AJ, Sims TJ, LeLous M, Bazin A: Collagen polymorphism in experimental granulation tissue. Biochem Biophys Res Comm 1975, 66: 1160-5. 